\title{
Störende Fettpolster einfach wegfrieren
}

Umfragen bei Patienten zufolge ist überflüssiges Körperfett - vor allem an sichtbaren Stellen - ein größeres kosmetisches Problem als Falten. Mit der Kryolipolyse ist inzwischen ein nicht invasives Verfahren zur Fettentfernung verfügbar. Die Dicke der behandelten subkutanen Fettschicht wird dabei selektiv reduziert, wie der Erfinder der Methode, Prof. Dieter Manstein von der US-amerikanischen Harvard Medical School, erläuterte. Für ihn ist die Methode „der Goldstandard der nicht invasiven Fettentfernung.“

Die mehrfach optimierte Technik mit der Bezeichnung CoolSculpting ${ }^{\circledR}$ basiert auf 15-jähriger Forschungs- und Ent- wicklungsarbeit und ist seit fünf Jahren in über 70 Ländern weltweit kommerziell verfügbar. Ein Basisgerät lässt sich - abhängig von der behandelten Körperregion wie Bauch, Oberschenkel, Flanken, Rücken und Achselbereich oder Doppelkinn - mit sechs verschiedenen Applikatoren kombinieren. Die Behandlung erfordert keine Anästhesie und hinterlässt keine Narben. Abhängig von der Ausdehnung des Areals können zwei Zyklen sinnvoll sein.

Dank umfassender Trainingsangebote und Marketingunterstützung des Herstellers - unter anderem mit der "CoolSculpting University“ - lässt sich die Me- thode optimal in die Praxis integrieren, ergänzte Dr. Jens Diedrichson, niedergelassener Dermatologe aus Düsseldorf. Er bietet die Methode seit 2014 an und hat damit besonders bei der Reduktion von Fettrollen, gut definierten Fettdepots und guter Hautelastizität sehr gute und langanhaltende Erfolge erzielt. Weniger geeignet sei die Methode zur Gewichtsabnahme sowie bei größen viszeralen Fettdepots. Frische Narben, Hauterkrankungen oder Wunden sollten jedoch ebenso wenig behandelt werden wie $\mathrm{Pa}$ tienten mit neuropathischen Erkrankungen oder implantierten Medizingeräten sowie Schwangere.

Dr. Andreas Häckel

Informationsveranstaltung „Erfahren Sie alles über das Original - Bodycontouring mit CoolSculpting ${ }^{\circledR}$, Frankfurt am Main, 3.6.2016; Veranstalter: Zeltiq

\section{Natürlichen Repair-Mechanismus unterstützen}

\section{Sonnenschutz für empfindliche Haut}

Mit Doctor Babor Repair Cellular Ultimate Protecting Balm SPF 50 steht ein Sonnenschutz für sensible Haut zur Verfügung. Der Balm schützt nicht nur vor schädigenden UV-A und UV-B-Strahlen. Er ist auch immer dann ein Must-Have, wenn die Haut besonders sonnenempfindlich ist - beispielsweise nach einer Mikro- dermabrasion oder Fruchtsäure-Peelings. Im Balm bieten UV-A und UV-B-Filter einen besonders effektiven Schutz vor schädigenden Sonnenstrahlen. Zusätzlich schützt ein sogenannter Cell Protect Complex mit einem intelligenten System: Die wirksamen Radikalfänger Vitamin E und C sind in eine Trägerkugel eingekap- selt, die sich bei UV-Bestrahlung öffnet. Dieser UV-Vektor funktioniert wie ein Sonnenschirm, der sich bei Sonneneinstrahlung automatisch aufklappt und die Zellen somit präzise schützt, wenn sie es benötigen. Gleichzeitig legt sich ein Kerzenstrauch-Extrakt wie ein Schutzmantel um die Zelle. Der Extrakt schützt die DNA der Zellen vor Schädigung durch UV-Strahlung und unterstützt den natürlichen Repair-Mechanismus der Haut.

Nach Informationen von Dr. Babor

Prävention und Therapie pathologischer Narben

\section{Unschöne Narben vermeiden}

Exzessive Bildung von Narbengewebe kann nach tiefen Verletzungen der Haut in Abhängigkeit von individueller Disposition, Lokalisation, chronischen Entzündungen, bei vermehrtem Zug auf die Wundränder oder verzögerter Wundheilung auftreten, erklärte PD Dr. Gerd Gauglitz, München.

Das in der täglichen Praxis häufig angewandte Narbentopikum Contractubex ${ }^{\circledast}$ mit Extractum Cepae, Heparin und Allantoin habe seine Wirksamkeit auf die Narbenheilung in verschiedenen Studien belegt, so Gauglitz. Der Zwiebelextrakt wirke antiproliferativ und antiinflam- matorisch auf Fibroblasten und hemme die in überschießenden Narben gesteigerte Kollagenproduktion. In einer prospektiven randomisierten Studie an 65 Kindern mit Operationen im Thoraxbereich konnte gezeigt werden, dass eine frühzeitige Behandlung (3 Wochen nach OP, $2 \times$ tgl.) mit dem Zwiebelextrakt in den ersten sechs Monaten zu deutlich kleineren Narben führte; überschießende Narben entwickelten sich seltener als in der Vergleichsgruppe ohne Behandlung. Eine Beobachtungsstudie an 1.268 Patienten ergab eine signifikante Verbesserung der Narbenqualität durch den Zwiebelex- trakt bei Patienten mit Zustand nach OP, Schnitt- und Schürfwunden sowie Verbrennungen. Die Anwendung des Narbengels wurde in die internationalen Leitlinien zur Behandlung von überschießenden Narben aufgenommen. Hier werde es für jüngere, hypertrophe Narben und zur Prävention bei Patienten mit erhöhtem Risiko der pathologischen Narbenentstehung empfohlen. Seit Kurzem steht mit dem Contractubex ${ }^{\circledast}$ Intensivpatch (Extractum Cepae, Allantoin) eine Narbentherapie für die Nacht mit okklusivem Effekt zur Verfügung.

Susanne Pickl

Mittagsseminar „Update Narbenprävention und Narbentherapie“, 25. Fortbildungswoche für praktische Dermatologie und Venerologie, München, 25.7.2016; Veranstalter: Merz Pharmaceuticals 